\title{
Next-generation sequencing for cancer drug development: the present and visions for the future
}

NGS for Cancer Drug Development

24-26 September 2013, Boston, MA, USA

Many of us who, prior to the -omics revolution, staunchly pursued the molecular basis of disease causation recognized the fundamental importance that biomarkers held for the eventuality of personalized medicine, more accurate diagnostics and targeted drug discovery. With slab sequencing apparatuses now mere memories relegated to the dusty reaches of old laboratory cabinets, we are fervently unraveling the complexities of many diseases through newer, more powerful innovative technologies. Quicker than ever before, we are achieving a deeper understanding of causative genetic lesions, perturbed pathways and the functions of altered networks. This has opened the door to a golden age of data generation that shows promise as a vital key to the successful treatment of human diseases. Hanson Wade recently organized a series of three related symposia that highlighted advances in industry and academic research in the areas of drug development, companion diagnostics and disease profiling: 'NGS for Cancer Drug Development' conference in Boston on 24-26 September 2013, the 'NGS Data Analysis' conference in San Francisco on 15-17 October 2013 and the 'World CDx' conference in Boston on 12-15 November 2013. These meetings provided forums for leaders to present advancements in personalized medicine, strategies for drug development, and innovations in diagnostics and other important areas. Their highly interactive nature also provided opportunities for industry and academic attendees to identify common hurdles that stand in the way of progress, (e.g., biomarker validation, analytical software limitations, data sharing, sample handling, regulatory and reimbursement restrictions), and to propose strategies for independent and community approaches toward overcoming such hurdles. In the interest of space, this review will be limited to the first in this series, the NGS for Cancer Drug Development conference, and its six major areas of focus.

Exploring the applications of nextgeneration sequencing technology in cancer drug development

Jadwiga Bienkowska, Biogen, spoke about work on the John Cunningham virus and progressive multifocal leukoencephalopathy. Dr Bienkowska stressed the importance of understanding the granular details of molecular profiling methodologies and how nextgeneration sequencing (NGS) results can be compromised. In providing examples of how specific experimental steps, such as PCR, can induce sequencing artifacts, she underscored the importance of undertaking stringent validation measures.

Andre Rosenthal, Signature Diagnostics, detailed a platform for ultra-deep sequencing of known cancer drivers detected in circulating free DNA from plasma of patients with colorectal cancer. He also presented a robust setup of prospective multicenter clinical trials in Germany to discover and validate bio-
Scott M Kahn

Dept. of Urology, Columbia University, NY, USA

and

Biomarkers Council, International Cancer Advocacy Network, Phoenix, AZ, USA and

Oncostem Biotherapeutics, LLC, NY, USA and

smk1@columbia.edu 
markers for early detection/screening and prognosis of colorectal and other cancers. Rosenthal brought up the point of limiting diagnostic testing to known, clinically relevant targets, while others debated the value of more comprehensive profiling strategies.

\section{Overcoming bioinformatic bottlenecks}

Dongliang Ge, Gilead Sciences, summarized bioinformatics strategies of NGS for cancer drug development, and the incorporation of biomarkers into clinical trial design. A common theme is that drug developers are increasingly turning to earlier reliance on biomarker data to guide decisions. Innovative bioinformatic algorithms, including specific platforms described at the subsequent World CDx conference, are currently being developed for improved defining of clinical trial design parameters.

\section{Technologies \& techniques for detecting structural variants from NGS data}

Yinghui Zhou, AVEO, presented a detailed genomic analysis of a tumor from an invasive and metastatic colorectal cancer model. Known and novel oncogenic driver events were identified, including amplified $C d k 6 / C d k 14$ and mutant $K$-ras, leading to the implementation of a novel combination regimen that completely eradicated metastasis to the lung. These systems could provide relevant insights into multiple targeted therapy options for humans.

Kalle Ojala, MediSapiens, highlighted the wideranging data integration platform OncoGenomics Explorer, touching upon its drug development-related applications, including the stratification of patients based on genetic profiles, identification of companion diagnostics (CDx) and pharmacological biomarkers, assessing the repurposing of existing compounds, and establishing connections between genetic and clinical events.

Thomas Westerling, Dana-Farber Cancer Institute, presented a generalized strategy for discovering noncoding single nucleotide variants that influence transcription factor binding. By measuring allelic bias in chromatin immunoprecipitation sequencing transcription factor-binding site interactions at heterozygous positions, Dr Westerling is identifying functionally relevant single nucleotide polymorphisms in breast cancer with the ultimate goal of identifying single nucleotide polymorphisms that are consequential to patient treatment.

Max van Min, Cergentis, provided examples of targeted, complete sequencing of the $A L K$ and $B R C A 1$ genes using Targeted Locus Amplification (TLA), a technology that enables complete amplification and hypothesis-neutral sequence generation for any gene using just one primer pair designed from the middle of the gene(s) of interest.

\section{Integrating NGS into clinical trial design \& practice}

Brian Dougherty, AstraZeneca, spoke on the stratification of clinical trials using biomarkers developed by NGS, stressing the importance of quality control. He included details of technical applications, including target enrichment strategies such as Agilent's Haloplex platform, and recent offerings for processing formalinfixed, paraffin-embedded (FFPE) samples. Technologies that improve the reliability of results from FFPE samples are extremely valuable for diagnostic approaches, considering the difficulties encountered in standardizing sample processing during multisite clinical studies, and the far-reaching consequences of processing delays on molecular profiling results - elegantly covered at the World CDx meeting by Sharon Moulis, Merrimack Pharmaceuticals.

Peter Kazon, American Clinical Laboratory Association, expertly tackled an increasingly complex and consequential topic - how advances in molecular diagnostics are leading to transformative changes at the Centers for Medicare \& Medicaid Services (CMS) and the US FDA. He detailed challenges in the areas of diagnostic coding and reimbursement, as well as in drug development. The newly unveiled payment schedules for molecular tests are not without controversies (e.g., nonreimbursement of predictive and diagnostic algorithms), and many issues still need to be addressed in the area of CDx testing, including the use of laboratory-developed tests, the expanding use of NGS assays and off-label drug use.

\section{Establishing the translational \& clinical potential of NGS in cancer drug development} George Mulligan, Millennium Pharmaceuticals, described the impact of specific mutations on Velcade ${ }^{\circledR}$ sensitivity in multiple myeloma cases. In a small cohort, K-Ras and N-Ras mutations appeared to be mutually exclusive events in multiple myeloma. Interestingly, only the presence of an $N$-Ras mutation (11/12 cases) was strongly predictive of nonresponsiveness to Velcade. These findings beg the question of what functional differences are imparted by $K$-Ras and $N$-Ras mutations and how they affect Velcade responsiveness. Follow-up profiling studies could shed light on such functionality.

Todd Lowe, Maverix Biomics, described how t-RNAderived miRNAs modulate cellular proliferation, and provided examples of associations between altered expression of specific species and certain cancers. In addition, Dr Lowe presented platforms that enable RNA 
profiling of exosomes, an area that is attracting increasing attention for the identification of disease-associated biomarkers.

Oscar Puig, Roche, offered a comprehensive illustration of the importance of data integration from a variety of approaches, including FACS, RNA sequencing (RNAseq), protein measurements in tumor and blood, and immunohistochemistry for the identification of predictive biomarkers for a monoclonal antibody used to treat liver cancer.

Diane Ilsley, Asuragen, presented applications of quantitative functional index PCR to targeted NGS. This platform enables analysis of FFPE samples, and fine-needle aspiration tumor biopsies preserved in a proprietary buffer, incorporating controls and quality control for each step.

Alexander Miron, Harvard/Covariance Biosciences, described a novel mutational detection methodology, exon grouping analysis. Exon grouping analysis utilizes a high-throughput, automated system to identify mutations by their formation of PCR-generated heteroduplexes and altered gel migration patterns.

Michael Vishnevetsky, Wafergen Biosystems spoke on targeted clinical NGS using the SmartChip TETM Target Enrichment System. Dr Vishnevetsky presented data that by using as little as $22 \mathrm{ng}$ of input genomic DNA, a targeted BRCA1/2 panel resulted in $100 \%$ coverage at $1000 \times$ with over $99.9 \%$ uniformity of coverage.

Ray Lee, Roche, offered examples of the role of biomarkers in the development process for three targeted lung cancer therapies, and their use in predicting drug responsiveness. Dr Lee also touched upon the use of biomarker data in making go/no-go decisions during different stages of drug development. In addition to points presented, well-designed software algorithms, such as those presented later during the World CDx conference have been developed to guide drug developers through difficult decision-making steps.

Danielle Greenawalt, AstraZeneca, discussed using publicly available NGS data for target discovery. For identifying potential cancer drivers from such data sets, she described the use of software such as DriverNet [1] and HotNet [2], along with Oncodrive CLUST [3] for the identification of mutational clusters. Current gaps in publicly available data sets include niche tumor types/populations, data on relapsed/resistant patients and results from targeted therapy trials, although some groups are addressing these and other issues. Adding to this synopsis, and it cannot be underscored enough, the presence of errors in all levels of public databases makes it imperative for individual investigators to take all appropriate measures to ensure the quality and reliability of data that are relied upon for mining purposes.

\section{Living the reality of personalized medicine}

The conference concluded with the author presenting a detailed overview of the work performed by the International Cancer Advocacy Network (ICAN) in support of cancer patients, and a lead advocacy issue being addressed by the ICAN Biomarkers Council - hastened development and rapid implementation of wholegenome/transcriptome sequencing (WGS/RNAseq) as a primary diagnostic test for late-stage cancer patients, and its reimbursement.

Founded in 1996, ICAN is a not-for-profit foundation that offers a patient-centric approach to personalized cancer management. ICAN works hand in hand with each cancer patient, carefully reviewing entire medical histories and supporting patients at each and every step so they can work with their clinical teams to obtain proper tests and optimize therapeutic regimens. ICAN has assisted over 7000 cancer patients, from all 50 states and over 50 countries throughout the world, with a strong focus on those with late-stage cancers. These efforts are backed by a world-class physician's council with collective expertise in all cancer types, a biomarkers council comprised of leaders from academia and industry, and supported by an extensive global network of dedicated volunteers. ICAN also works feverishly on the local and national governmental levels advocating for legislation of benefit to cancer patients.

Since its earliest days, ICAN has recognized the clinical importance of molecular profiling to patient care. Given that over 500,000 patients per year succumb from cancer in the USA, ICAN is a strong advocate for the development of WGS/RNAseq as a primary diagnostic test for late-stage cancer patients. Early reports on genomic sequencing-based strategies $[4,5]$ are encouraging, demonstrating their utility to identify clinically actionable therapies that would have otherwise been overlooked by using more restrictive diagnostic tests for cancer patients. Thus, there is great promise for these technologies to help extend or save the lives of thousands of patients each year in the USA. ICAN is therefore calling upon governmental officials in decision-making capacities to support the fastest possible development of WGS/RNAseq as a reliable, costeffective diagnostic testing platform for late-stage cancer patients, and to actively lead all efforts to streamline clinical implementation and reimbursement.

\section{The future: cancer prevention, improved diagnostics, novel approaches \& funding requirements}

Taken together, these meetings provided valuable interactive venues for the dissemination of state-ofthe-art approaches to drug development and diag- 
nostics, and the sharing of visions. It is inspiring to reflect upon the progress in the fields of diagnostics and personalized medicine that have been made in the past decade, which is a proud tribute to the collective innovative nature of those who work in these fields. However, even though personalized medicine is closer to mainstream than ever, we are still faced with hurdles that need to be overcome. It is sobering that while effective in certain leukemias, the efficacy of singletargeted therapies in extending the lives of patients with solid tumors has been relatively disappointing. Much hope is now placed on the promise of multipletargeted therapy regimens to head off the ability of cancer cells to reprogram themselves and escape killing by single agents. Looking beyond targeting cancer drivers, we need to fully exploit networking analysis to identify overstressed bottlenecks in cancer pathways that may not be mutated themselves, but may present as attractive druggable targets. For example, at the NGS Data Analysis meeting Debasish Raha presented a role for ALDH in drug resistance. Similar studies could greatly enhance our arsenal of cancer drugs. We also need to better understand interactions that occur within the tumor microenvironment, and how the signaling within individual cancer cells is modulated through interactions with neighboring cells and the intercellular millieu. Also, we need to rediscover cancer prevention through the eyes of -omics platforms: how nutrition, prophylactic drugs, the microbiome, the environment and other sources can be turned in one's favor. Along with this is the need

\section{References}

1 Shah Lab for Computational Cancer Biology. DRIVERNET. http://compbio.bccrc.ca/software/drivernet

2 Raphael Lab. HotNet. http://compbio.cs.brown.edu/projects/hotnet

3 Biomedical Genomics. OncodriveCLUST. http://bg.upf.edu/group/projects/oncodrive-clust.php for further development of technologies to detect the earliest stages of disease (e.g., circulating tumor cell detection and soluble DNA) so that our collections of targeted therapies may be put to more effective use at earlier time points. Finally, I conclude with funding issues. A common theme throughout these three meetings is the lack of available funding at a time when we are making historical inroads into disease causation and treatment. We need study sections, federal funding agencies and industry to continue to actively seek out innovative ideas, both hypothesis driven and nonhypothesis driven, as opposed to adopting more conservative approaches in the face of budgetary constraints. The quicker we can implement widespread personalized approaches to disease treatment, more efficaciously treat complex diseases and improve upon the clinical relevance of diagnostic testing, the sooner we can raise critical funding by rendering current laboratory hardware obsolete and selling it along with our old slab gel apparatuses as valuable historical museum pieces.

\section{Financial \& competing interests disclosure}

The author has no relevant affiliations or financial involvement with any organization or entity with a financial interest in or financial conflict with the subject matter or materials discussed in the manuscript. This includes employment, consultancies, honoraria, stock ownership or options, expert testimony, grants or patents received or pending, or royalties.

No writing assistance was utilized in the production of this manuscript.

4 Weiss GJ, Liang WS, Demeure MJ et al. A pilot study using next-generation sequencing in advanced cancers: feasibility and challenges. PLoS ONE 8(10), e76438 (2013).

5 Frampton GM, Fichtenholtz A, Otto GA et al. Development and validation of a clinical cancer genomic profiling test based on massively parallel DNA sequencing. Nat. Biotechnol. 31(11), 1023-1031 (2013) 